\title{
A compararison of magnetization transfer methods to assess brain and cervical cord microstructure in multiple sclerosis
}

Alfonso Lema ${ }^{1}$, Courtney Bishop ${ }^{2}$, Omar Malik ${ }^{1}$, Miriam Mattoscio ${ }^{1}$, Rehiana Ali $^{1}$, Richard Nicholas $^{1}$, Paolo A Muraro ${ }^{1}$, Paul M Matthews ${ }^{1 *}$, Adam D Waldman ${ }^{3 *}$, Rexford D Newbould $^{2 *}$

${ }^{1}$ Division of Brain Sciences, Department of Medicine, Imperial College London

${ }^{2}$ Imanova Centre for Imaging Sciences, London

${ }^{3}$ Department of Imaging, Imperial College Healthcare NHS Trust

*Joint senior authors

Running Title: MT of brain and c-spine in MS

Keywords: Magnetization Transfer; MRI; Multiple Sclerosis; Atrophy; Spinal Cord

*Correspondence to:

Prof. Paul M. Matthews

Edmond and Lilly Safra Chair

Head, Division of Brain Sciences,

Imperial College London

Burlington Danes Building

DuCane Road, London WC12 0NN

Tel: 00442075942612 
Acknowledgements:

This work was supported by the Imperial College Healthcare Trust Biomedical Research Centre, Medical Research Council (Ref. No. G0800679), GlaxoSmithKline and the EC. PMM acknowledges personal support from the Edmond J Safra Foundation and Lily Safra.

Disclosure statements:

Dr Muraro has received travel support and speaker honoraria from Bayer HealthCare, Bayer Pharma, Biogen Idec, Merck-Serono, Sanofi Aventis and Novartis.

Prof. Matthews and Dr. Newbould were employees of GlaxoSmithKline Research and Development at the time of the study. Prof. Matthews has received research or educational funding or has had honoraria paid to Imperial College from Biogen, Novartis, IXICO and Transparency Life Sciences

Prof Waldman has received educational grant funding or speaker honoraria from Bayer HealthCare, Biogen and Novartis. 


\section{Abstract}

Background: Demyelination is a core pathological feature of multiple sclerosis (MS) and spontaneous remyelination appears to be an important mechanism for repair in the disease. Magnetization transfer ratio imaging (MTR) has been used extensively to evaluate demyelination, although limitations to its specificity are recognized. MT saturation imaging (MTsat) removes some of the T1 dependence of MTR. We have performed a comparative evaluation of MTR and MTsat imaging in a mixed group of subjects with active MS, to explore their relative sensitivity to pathology relevant to explaining clinical outcomes.

Methods: 134 subjects underwent MRI of their brain and cervical spinal cord. Isotropic 3D pre- and post-contrast T1-weighted and T2-weighted fluid-attenuated inversion recovery (FLAIR) volumes were segmented into brain normal appearing white matter (NAWM), brain white matter lesions (WML), normal appearing spinal cord (NASC) and spinal cord lesions (SCL). Volumes and metrics for MTR and MTsat histograms were calculated for each region.

Results: Significant Spearman correlations were found with the Expanded Disability Status Scale (EDSS) and timed 25-foot walk (T25FW) for the whole brain and WML MTR, but not in that from the NAWM or any cervical spinal cord region. By contrast, the MTsat was correlated with both disability metrics in all these regions in both the brain and spine.

Conclusions: This study extends prior work relating atrophy and lesion load with disability, by characterization of MTsat parameters. MTsat is practical in routine clinical applications and may be more sensitive to tissue damage than MTR for both brain and cervical spinal cord. 


\section{Introduction}

Multiple sclerosis (MS) produces both inflammatory and degenerative pathology in the brain and spinal cord. Conventional magnetic resonance imaging (MRI) of the brain is highly sensitive for detecting focal inflammatory lesions in patients with MS. Assessments of the burden of brain T2 lesions are used to monitor evolution of MS clinically. However, only modest correlations between brain MRI lesion load and clinical disability have been found. ${ }^{1}$ One explanation for this is that comprehensive detection of lesions in spinal cord MRI still is more challenging than for the brain although occurrence of MS lesions in the cord is believed to give rise to clinical symptoms more often than lesions located in the brain. ${ }^{2,3}$ The assessment of MS pathology using T2 weighted images also is limited by the poor specificity of this technique to the heterogeneous substrates of MS lesions, ${ }^{4}$ and by its inability to provide information about the microscopic changes occurring in the normal appearing white matter (NAWM). ${ }^{5}$ Perhaps most important, however, is that substantial neuronal and axonal loss are found in the brain and spinal cord, in addition to demyelination. ${ }^{6-10}$

Magnetisation transfer imaging (MTI) provides quantitative metrics sensitive to microstructural changes reflecting some neurodegenerative aspects of MS pathology which, ${ }^{11,12}$ in conjunction with conventional MRI measures, allow the combined macroscopic and microscopic disease burdens to be estimated. ${ }^{13}$ More specifically, MTI reflects the exchange of magnetization between freely mobile protons and those associated with macromolecules in myelin or axonal membranes. ${ }^{14}$ The MT ratio (MTR) is strongly correlated with the percentage of residual myelin and axons present in MS lesions and NAWM. ${ }^{15}$ However, conventional MTR is sensitive to biophysical parameters that may limit its precision, particularly in the spinal cord. 
The alternative approach of quantitative MT saturation (MTsat) offers a measure that, unlike MTR, is minimally affected by $\mathrm{T} 1$ relaxation and is less sensitive to B1 inhomogeneities. White matter (WM) to grey matter (GM) contrast in MTsat maps is increased relative to that in MTR maps. ${ }^{16}$ MTsat thus may enhance insights into clinicopathological correlations related to changes in the cervical cord in MS relative to the MTR.

The goals of this study are to compare MTR and MTsat measures across an MS population and to analyse their capability to discriminate between disability levels when they are applied to the brain and cervical spinal cord of a cohort of 134 patients. Correlations between MTR and MTsat histogram metrics of the brain and the cervical cord and disability were explored.

\section{Methods}

Patients

The cohort of patients formed part of the Patient Research Cohort Rapidly Evolving Multiple Sclerosis Study (PRC - REMS), which had the approval of the London - Chelsea Regional Ethics Committee (NCT01044576) and was conducted in accordance with the principles of Good Clinical Practice (GCP). MRI scans of the brain and cervical spine for 134 patients were available for this study. The degree of disability was estimated according to the Kurtzke's Expanded Disability Status Scale (EDSS) and the Timed 25-Foot Walk (T25FW) scale from the Multiple Sclerosis Functional Composite.

\section{MRI acquisition}

The study was performed on a 3T clinical MR system (Magnetom Verio, Siemens Medical Solutions, Erlangen, Germany). A combined head and neck RF coil system consisting of a twelve channel phased array head coil and four channel phased array neck coil 
were used for reception. The system body coil was used for all excitation and MT RF pulses, and no repositioning occurred between sequences. Pre and post contrast T1-weighted 3D MPRAGE volumes were acquired similarly to the ADNI recommended parameters except 1 mm isotropic resolution, ${ }^{17}$ parallel imaging (PI) factor of 2 and 256x192 mm field of view (FOV) in 5m: 21s in the brain. A 0.6x0.6x2.0mm resolution and 160x160x160mm FOV was used in the cervical spinal cord in 3m:43s. A physician injected gadolinium contrast (Gadoterate meglumine, Dotarem, Guerbet, $0.1 \mathrm{mmol} / \mathrm{kg}$ ) 5 minutes before the acquisition of the final image volumes: the post contrast MPRAGE volumes of the brain and c-spine. All other images were acquired before the contrast injection. T2-weighted Fluid Attenuated Inversion Recovery (FLAIR) volumes were acquired with $1 \mathrm{~mm}$ isotropic resolution and 160 sagittal sections in a single 3D slab, 250x250 mm FOV, PI factor of 2, inversion time (TI) $1800 \mathrm{~ms}$, repetition time (TR) 5s, and echo time (TE) $395 \mathrm{~ms}$, in 5m: 52s in the brain. A 3D FLASH sequence with $1 \mathrm{~mm}$ isotropic resolution, 192 sagittal partitions, 256*240 FOV was employed in the brain (with a readout in the head-feet direction with $600 \mathrm{~Hz} /$ pixel bandwidth to reduce susceptibility artifacts). Six monopolar gradient echoes equally spaced between TE of 1.95 and $11.7 \mathrm{~ms}$ were acquired during a TR of $27 \mathrm{~ms}$. A PI factor of 2 was used for the GRAPPA parallel acquisitions. A flip angle of $5^{\circ}$ was chosen for one of the proton densityweighted (PD-w) dataset meanwhile another was MT-weighted by a band selective RF pulse of Gaussian shape with a duration of $12.24 \mathrm{~ms}, 2.2 \mathrm{kHz}$ frequency offset and nominal $540^{\circ}$ flip angle, in 7m: 20s. A T1-w volume, similar to the PD-weighted but with a TR of $15 \mathrm{~ms}$ and a flip angle of $18^{\circ}$ was also acquired in 4m: 05s. A similar set of three contrasts at $1 \mathrm{~mm}$ isotropic resolution were acquired in the cervical spinal cord, but with a smaller FOV of 192x156x120mm. The PD-w and MT-weighted acquisitions used a TR of 26ms, requiring 4m:59s each. The T1-w volume used a TR of $13 \mathrm{~ms}$ and flip angle of $15^{\circ}$. Other parameters remained the same as the brain acquisitions. 
Data Analysis

MTR maps were produced on a voxel by voxel basis according to the equation:

$$
M T R=100 \frac{M_{0}-M_{S}}{M_{0}}
$$

Where $M_{S}$ and $M_{0}$ are signal intensities with application of saturation pulse and without respectively. MTR values are reported in percent units (p.u). The MTsat parameter was calculated by inserting the estimated $A_{a p p}$ and $T_{1 a p p}$ values in the approximate signal equation for the MT FLASH experiment. ${ }^{18}$ The MTsat parameter represents the additional percentage reduction in the steady state FLASH signal caused by the saturation effect of a single MT pulse. It is calculated from this equation:

$$
\operatorname{MTon}_{P D w}=A_{a p p} \alpha\left(T R / T_{1 a p p}\right) /\left(T R / T_{1 a p p}+\alpha^{2} / 2+M T s a t\right)
$$

Using $A_{a p p}$ and $T_{1 a p p}$ obtained from these equations:

$$
\begin{gathered}
A_{a p p}=\frac{\left(2 T R_{P D w} / \alpha_{P D w}^{2}-2 T R_{T 1 w} / \alpha_{T 1 w}^{2}\right)}{\frac{2 T R_{P D w}}{\alpha_{P D w^{M T o f f}}-\frac{2 T R_{T 1 w}}{\alpha_{T 1 w} M T o f f_{T 1 w}}}} \\
T_{1 a p p}=\frac{\left(M T o f f_{P D w} / \alpha_{P D w}-M T o f f_{T 1 w} / \alpha_{T 1 w}\right)}{M T o f f_{P D w} \alpha_{P D w} / 2 T R_{P D w}}-M T o f f_{T 1 w} \alpha_{T 1 w} / 2 T R_{T 1 w}
\end{gathered}
$$

MTsat has been shown to be insensitive to inhomogeneities in RF transmit field and receive fields.(16) Unlike MTR, the MTsat is not affected by T1 relaxation.

SIENAX from FSL was used on T1-w images in the extraction of the brain and segmentation of GM and WM using a partial volume of threshold of 0.70 and estimation of their volumes. ${ }^{19}$ The cervical cord, brain WM lesions, and cervical spinal cord lesions were 
extracted manually with Analyze 11.0 (AnalyzeDirect/Mayo Clinic, KS). For each region, intensity distribution maps for segmented MTsat and MTR were calculated by Matlab (Mathworks Natick, MA) and histograms were generated for each subject with bins of width 0.25 percent units (p.u.). ${ }^{20}$ The histogram median and height of the highest peak were recorded. Linear co-registration of datasets was performed using FLIRT. ${ }^{21}$

Volumes were normalized with intracranial volume (ICV) to reduce inter-individual variation. Inter-subjects coefficients of variation $(\mathrm{CoV})$ were calculated:

$$
\mathrm{CoV}=\mathrm{SD} / \text { mean } \times 100 \%
$$

Spearman rank-order correlation coefficient and Spearman's partial correlations (controlling for age, gender and disease duration) assessed associations between volumes, MTR and MTsat histogram median and peak height, and both EDSS and T25FW.

Mixed models with patient as a random factor were calculated after the imputation of missing values. They determined the correlation between magnetization transfer histogram metrics and the volumes (segmented lesions, brain and cord) with disability scales EDSS and T25FW. Parameter estimates and marginal means with 95\% confidence intervals were presented.

A two tailed p value less than 0.05 was considered significant and corrected thresholds for $\mathrm{p}$ were applied for multiple comparisons. All statistical analyses were performed with SPSS for Windows (v. 17, SPSS Inc., Chicago, Illinois) except the comparison of correlations, which was done with the cocor package. ${ }^{22}$

\section{Results}

Subjects included people with both relapsing-remitting MS (RRMS) and secondary progressive MS (SPMS) (Table 1). T1 lesions or T2 hyperintensities in the FLAIR volumes 
were identified in 121 cases. Cervical T2 lesions were identified in 19 subjects. 121/123 datasets met blinded image QC criteria.

Manual spinal cord segmentation was performed in fifty-two subjects. The MTsat maps showed higher contrast between WM, GM and cerebrospinal fluid (CSF) relative to the MTR maps. The average histograms for the whole brain and for WM, GM and CSF for the cohort of patients, illustrate this (Figure 1a and 1b); the MTR histogram did not show a clear mode for the CSF. The MTsat peak height in the brain parenchyma was correlated with the MTR peak height $(r=0.590 ; p<0.001)$. This was not found for CSF $(r=0.122 ; p=0.188)$. Segmentation of the spinal cord into white and grey matter was not performed for tissue specific analyses in the spinal cord.

Both whole brain MTR and MTsat were correlated with median WML volumes ( $\mathrm{r}=$ 0.654, $\mathrm{p}<0.001$ for MTR and $\mathrm{r}=0.491, \mathrm{p}<0.001$ for MTsat), and their comparison showed they were significantly different $(\mathrm{z}=2.892 ; \mathrm{p}=0.004)$. Significant correlations also were found between both magnetization transfer and clinical disability measures (EDSS and T25FW) (Table 2). For MTsat, significant correlations were found between median values in NAWM and both EDSS and T25FW. No significant disability correlations were found for median NAWM MTR.

Significant correlations were found between the median MTsat and EDSS for all cervical spinal cord regions (Table 3). No significant correlations were found between median MTR values and either the EDSS or T25FW.

Correlations between MTR and MTsat histogram metrics (median values, peak heights and location) for the whole brain and either NAWM and the whole cervical spinal cord or the normal appearing spinal cord (NASC) were explored. No correlations were found between MTR or MTsat metrics obtained from the brain and the cervical cord although, we found a trend towards a significant correlation (i.e., those that reached $\mathrm{p}<0.017$, Bonferroni 
corrected) between the median whole brain MTsat and peak height of the whole cervical cord MTsat when controlling for age $(r=0.348 ; \mathrm{p}=0.024)$.

Mixed model analyses revealed that median and peak height MTsat explained significant amounts of disability measures. In a model with the following variables: age, gender and metrics (median and peak height) for the whole brain and c-spine, both the median whole brain MTsat $(\beta=-0.22[-0.43 ;-0.01], \mathrm{p}=0.041)$ and peak height of the whole cervical spinal cord MTsat $(\beta=-17.11[-32.84 ;-1.38], \mathrm{p}=0.033)$ showed significant associations with EDSS. The mixed model with age, gender, histogram metrics (median whole brain and c-spine) and region volumes (segmented lesions, brain and cord) as independent variables showed significant associations for the median whole brain MTsat $(\beta=$ $-0.30[-0.59 ;-0.01], \mathrm{p}=0.047)$ and median whole cervical spinal cord MTsat $(\beta=-0.21[-$ 0.35;-0.06], $\mathrm{p}=0.006$ ) with T25FW.

The mixed models did not provide evidence that whole brain volume or individual (GM or WM) tissue volume fraction measures explained a significant proportion of the disability. Spearman rank-order correlations for T2 lesion volume $(r=-0.254 ; p=0.005)$, white matter $(r=0.181 ; p=0.048)$ and grey matter $(r=-0.189 ; p=0.038)$ fractions with EDSS.

\section{Discussion}

Magnetisation transfer provides a potentially useful complement to conventional MRI in assessment of patients with MS. ${ }^{23}$ Several studies have shown that MTR values in NAWM are altered in all of the major phenotypes of MS. Reduced MTR values have been found in NAWM, even in patients with clinically definite MS and very few or no T2 visible lesions. ${ }^{24}$ Quantitative magnetisation transfer, qMT, allows parameters which reflect myelin integrity to be estimated independently of $\mathrm{T} 1$ and cross-relaxation rates between bound and unbound 
proton fractions. ${ }^{25}$ However, although qMT has a higher specificity for pathological processes underlying MS and is independent of MRI sequence, it requires long acquisition times and complex post-processing, which have limited its application to animal models, post mortem and experimental phase human imaging. ${ }^{26,27}$

MTR provides a measure reflecting microstructural information. ${ }^{15,20}$ In general, MS pathology is associated with a decrease in MTR: low MTR has been found in lesions that were not apparent on conventional MRI. Myelin loss (and, to a smaller extent, oedema) reduces MTR values in $\mathrm{MS} .^{21}$ Several studies have shown that MTR histogram derived measures of the cervical cord correlate well with the clinical manifestations of MS and are related to demyelination. ${ }^{23,28}$ Our cohort had by definition a low to mid-level EDSS, with a maximum EDSS of 6.0. Other studies have included subjects with higher EDSS, which may explain our failure to identify a correlation between NAWM MTR and disability in this population.

Previous studies found T2 lesion volume in the brain or spinal cord have not tended to show strong or significant correlations with the EDSS, ${ }^{1,29,30}$ although, in our population, there was a significant Spearman significant correlation of the brain T2 lesion volume, white matter and grey matter fractions with EDSS. By contrast, we did not find simple correlation between cord volumes with EDSS and T25FW. Failure to identify some associations reported by others may reflect the restricted range of our sample of patients, who were mildly disabled (median EDSS = 3.5), with a well preserved ambulatory function and shorter disease duration (median disease duration $=2$ years). Studies which involved larger cohorts with a longer disease duration ( $>20$ years) and higher EDSS (median of 4, range 0-8) have reported associations between upper spinal cord cross sectional area and disability. ${ }^{31}$

No significant relationships between brain and cervical cord volumes and MTR/MTsat metrics were found and this suggest a non-uniform disease process in MS. The 
processes may affect each region independently or show differential involvement between patients. In the brain tissue, axonal transection has been documented at the center and the periphery of demyelinating lesions leading to axonal and tissue volume loss in the adjacent WM, but in a large cross sectional MRI study and in a post mortem study no relationship between spinal cord atrophy and brain lesions was found. ${ }^{28,32,33}$

We hypothesised that MTsat measures, which take into account biophysical variations that may reduce the precision of MTR, may enhance the potential for discovery of clinicpathological correlations related to magnetisation transfer. Consistent with this, we found significant correlations between median NAWM MTsat and EDSS and T25FW that were not found for MTR. Similar results were found for the cervical spinal cord, which also showed a correlation between median MTsat values and EDSS, although the same relationship was not found for MTR.

This apparent difference in sensitivity is likely to reflect differences between the two acquisition sequences to biophysical properties of tissue. A major contribution to decreased magnetisation transfer in MS arises from reduced myelin volume, although increased tissue water also reduces MTR values in MS through effects on regional T1. ${ }^{32}$ Lower axon density (which can vary between people with MS independently of differences in myelin density) also increases tissue T1 recovery time, and can itself decrease MTR. ${ }^{34}$ MTsat is relatively insensitive to T1 dependent effects, which are a significant source of variance in the MTR signal; this is likely to account for the greater contrast between tissue compartments seen with MTsat in the population observed here. The significantly lower correlation between MTsat and white matter lesion size versus MTR and lesion size that we observed this provide indirect evidence that MTsat is less dependent than MTR on lesion volumes, suggesting that it reflects microstructural damage outside of lesions to a greater extent. 
Other studies are necessary to explore the limitations or advantages of MTsat versus conventional MT imaging in MS, preferably with a gold standard for comparison, such as histology from post mortem tissue. This could allow a direct determination of whether the MTsat technique was more sensitive to underlying axonal or cellular loss. Visual inspection of histograms noted that GM, WM, and CSF were much more separated in the MTsat versus MTR histograms. Similarly, the whole cervical spinal cord MTR histogram was unimodal while that of MTsat was bimodal. A full comparison of the ability of MTR versus MTsat to discriminate tissue type needs to be performed.

The absence of a healthy control cohort is a major limitation of our study. Furthermore, no longitudinal data is available. Comparisons can only be made within the diseased group at a single timepoint. A full study tracking both healthy and MS subjects over time is the next logical step. The manual segmentations in the spinal cord were only performed by a single researcher due to the high labour required; no metrics of inter-reader reliability are available.

In conclusion, MTsat discriminated between disability levels in MS better than MTR in the NAWM and in the spinal cord in this study population of low to moderate EDSS and short disease duration. We speculate that MTsat may be more sensitive to microstructural tissue damage in MS than is MTR, and may prove useful and practicable for imaging in larger scale clinical studies and therapeutic trials.

\section{References}

1. Goodin DS. Magnetic resonance imaging as a surrogate outcome measure of disability in multiple sclerosis: Have we been overly harsh in our assessment? Ann Neurol. 2006;59(4):597-605.

2. Rovira A, Tintoré M. Spinal cord MRI should always be performed in clinically 
isolated syndrome patients: No. Mult Scler J . 2014 Nov 1;20 (13 ):1686-7.

3. Tartaglino LM, Friedman DP, Flanders AE, Lublin FD, Knobler RL, Liem M. Multiple sclerosis in the spinal cord: MR appearance and correlation with clinical parameters. Radiology. 1995;195(3):725-32.

4. Filippi M. Non-conventional MR techniques to monitor the evolution of multiple sclerosis. Neurol Sci. 2001;22(2):195-200.

5. Barbosa S, Blumhardt LD, Roberts N, Lock T, Edwards RHTHT. Magnetic resonance relaxation time mapping in multiple sclerosis: Normal appearing white matter and the “invisible” lesion load. Magn Reson Imaging. 1994;12(1):33-42.

6. DeLuca GC, Ebers GC, Esiri MM. Axonal loss in multiple sclerosis: A pathological survey of the corticospinal and sensory tracts. Brain. 2004;127(5):1009-18.

7. Evangelou N, DeLuca GC, Owens T, Esiri MM. Pathological study of spinal cord atrophy in multiple sclerosis suggests limited role of local lesions. Brain. 2005;128(Pt 1):29-34.

8. Gilmore CP, DeLuca GC, Bö L, et al. Spinal cord neuronal pathology in multiple sclerosis. Brain Pathol. 2009;19(4):642-9.

9. Peterson JW, Bö L, Mörk S, et al. Transected neurites, apoptotic neurons, and reduced inflammation in cortical multiple sclerosis lesions. Ann Neurol. 2001;50(3):389-400.

10. Vogt J, Paul F, Aktas O, et al. Lower motor neuron loss in multiple sclerosis and experimental autoimmune encephalomyelitis. Ann Neurol. 2009;66(3):310-22.

11. Dousset V, Brochet B, Vital A, et al. Lysolecithin-induced demyelination in primates: Preliminary in vivo study with MR and magnetization transfer. Am J Neuroradiol. 1995;16(2):225-31.

12. Dousset V, Grossman RI, Ramer KN, et al. Experimental allergic encephalomyelitis and multiple sclerosis: lesion characterization with magnetization transfer imaging. 
Radiology. 1992;182(2):483-91.

13. Filippi M, Campi a, Dousset V, et al. A magnetization transfer imaging study of normal-appearing white matter in multiple sclerosis. Neurology. 1995;45(3 Pt 1):47882.

14. Tofts PS, Steens SC a, van Buchem MA. MT: Magnetization Transfer. Quantitative MRI of the Brain. 2003. 257-298 p.

15. Van Waesberghe JHTM, Kamphorst W, De Groot CJ a, et al. Axonal loss in multiple sclerosis lesions: Magnetic resonance imaging insights into substrates of disability. Ann Neurol. 1999;46(5):747-54.

16. Helms G, Dathe H, Kallenberg K, Dechent P. High-resolution maps of magnetization transfer with inherent correction for RF inhomogeneity and T1 relaxation obtained from 3D FLASH MRI. Magn Reson Med. 2008;60(6):1396-407.

17. Jack CR, Bernstein MA, Fox NC, et al. The Alzheimer's Disease Neuroimaging Initiative (ADNI): MRI methods. Journal of Magnetic Resonance Imaging. 2008. p. 685-91.

18. Helms G, Dathe H, Dechent P. Quantitative FLASH MRI at 3T using a rational approximation of the Ernst equation. Magn Reson Med. 2008;59(3):667-72.

19. Smith SM, Zhang Y, Jenkinson M, et al. Accurate, Robust, and Automated Longitudinal and Cross-Sectional Brain Change Analysis. Neuroimage. 2002;17(1):479-89.

20. van Buchem M a, McGowan JC, Kolson DL, Polansky M, Grossman RI. Quantitative volumetric magnetization transfer analysis in multiple sclerosis: estimation of macroscopic and microscopic disease burden. Magn Reson Med. 1996;36(5):632-6.

21. Jenkinson M, Bannister P, Brady M, Smith S. Improved optimization for the robust and accurate linear registration and motion correction of brain images. Neuroimage. 
2002;17(2):825-41.

22. Diedenhofen B, Musch J. Cocor: A comprehensive solution for the statistical comparison of correlations. PLoS One. 2015;10(4).

23. Rovaris M, Filippi M. The value of new magnetic resonance techniques in multiple sclerosis. CurrOpinNeurol. 2000;13(3):249-54.

24. De Stefano N, Narayanan S, Francis SJ, et al. Diffuse axonal and tissue injury in patients with multiple sclerosis with low cerebral lesion load and no disability. Arch Neurol. 2002;59(10):1565-71.

25. Morrison C, Henkelman RM. A model for magnetization transfer in tissues. Magn Reson Med. 1995;33(4):475-82.

26. Schmierer K, Tozer DJ, Scaravilli F, et al. Quantitative magnetization transfer imaging in postmortem multiple sclerosis brain. J Magn Reson Imaging. 2007;26(1):41-51.

27. Dortch RD, Moore J, Li K, Jankiewicz M, et al. Quantitative magnetization transfer imaging of human brain at 7T. Neuroimage. 2013;64(1):640-9.

28. Lycklama a Nijeholt GJ, Van Walderveen MAA, Castelijns J a., et al. Brain and spinal cord abnormalities in multiple sclerosis: Correlation between MRI parameters, clinical subtypes and symptoms. Brain. 1998;121(4):687-97.

29. Gass A, Barker GJ, Kidd D, et al. Correlation of magnetization transfer ratio with clinical disability in multiple sclerosis. Ann Neurol. 1994;36(1):62-7.

30. Barkhof F. The clinico-radiological paradox in multiple sclerosis revisited. Curr Opin Neurol. 2002;15(3):239-45.

31. Kearney H, Rocca M a, Valsasina P, et al. Magnetic resonance imaging correlates of physical disability in relapse onset multiple sclerosis of long disease duration. Mult Scler. 2014;20(1):72-80.

32. Schmierer K, Scaravilli F, Altmann DR, Barker GJ, Miller DH. Magnetization transfer 
ratio and myelin in postmortem multiple sclerosis brain. Ann Neurol. 2004;56(3):40715.

33. Filippi M, Bozzali M, Horsfield $\mathrm{M}$ a, et al. A conventional and magnetization transfer MRI study of the cervical cord in patients with MS. Neurology. 2000;54(1):207-13.

34. Bergers E, Bot JCJ, De Groot CJ a, Polman CH, Lycklama à Nijeholt GJ, Castelijns J a, et al. Axonal damage in the spinal cord of MS patients occurs largely independent of T2 MRI lesions. Neurology. 2002;59(11):1766-71. 


\section{Tables}

Table 1: Summary of clinical characteristics of patients involved in the study.

\begin{tabular}{|c|c|c|c|}
\hline & All & Brain & C-Spine \\
\hline Subjects & 134 & 121 & 52 \\
\hline RRMS & 128 & 115 & 49 \\
\hline SPMS & 6 & 6 & 3 \\
\hline Age (years) & 39 & 39 & 38 \\
\hline Gender (F/M) & $107 / 27$ & $97 / 24$ & $41 / 11$ \\
\hline Disease Duration (years) & $3(0-16)$ & $3(0-16)$ & $2(0-13)$ \\
\hline EDSS & $3.5(2.0-6.0)$ & $3.5(2.0-6.0)$ & $3.5(2.0-6.0)$ \\
\hline T25FW (seconds) & $6.0(1.3-15.3)$ & $5.9(1.3-15.3)$ & $6.1(4.1-14.1)$ \\
\hline Relapses within last year & $2(0-5)$ & $2(0-5)$ & $2(0-4)$ \\
\hline
\end{tabular}

Median (range) values are shown for: age, disease duration, EDSS, T25FW and relapses within last year.

Abbreviations: RRMS = relapsing reemitting multiple sclerosis, SPMS = secondary progressive multiple sclerosis, EDSS = Expanded Disability Status Scale, T25FW = timed 25-foot walk. 
Table 2: Spearman rank-order correlation coefficients with both disability scales for MTR and MTsat in the brain ( ${ }^{*} \mathrm{p}<0.05$, corrected for multiple comparisons).

\begin{tabular}{|c|c|c|c|c|}
\hline & \multicolumn{2}{|c|}{ EDSS } & \multicolumn{2}{|c|}{ T25FW } \\
\hline MTR & $\mathrm{r}$ & p Value & $r$ & p Value \\
\hline Median whole brain & -0.271 & $0.003 *$ & -0.263 & $0.004^{*}$ \\
\hline Median NAWM & -0.178 & 0.051 & -0.170 & 0.062 \\
\hline Median WML & -0.264 & $0.003 *$ & -0.328 & $<0.001^{*}$ \\
\hline Peak height whole brain & 0.033 & 0.719 & 0.022 & 0.807 \\
\hline Peak height NAWM & -0.042 & 0.646 & -0.118 & 0.196 \\
\hline Peak height WML & -0.101 & 0.273 & -0.209 & $0.021^{*}$ \\
\hline MTsat & $r$ & p Value & $r$ & p Value \\
\hline Median whole brain & -0.288 & $0.001 *$ & -0.225 & $0.013^{*}$ \\
\hline Median NAWM & -0.238 & $0.008^{*}$ & -0.180 & $0.048^{*}$ \\
\hline Median WML & -0.230 & $0.011^{*}$ & -0.251 & $0.005^{*}$ \\
\hline Peak height whole brain & 0.014 & 0.882 & 0.140 & 0.125 \\
\hline Peak height NAWM & -0.011 & 0.905 & -0.004 & 0.964 \\
\hline Peak height WML & -0.017 & 0.852 & -0.014 & 0.878 \\
\hline
\end{tabular}

Abbreviations: EDSS = Expanded Disability Status Scale, T25FW = timed 25-foot walk, MTR = magnetization transfer ratio, MTsat = magnetization transfer saturation, NAWM = brain normal appearing white matter, WML = brain white matter lesion volume. 
Table 3: Spearman rank-order correlation with both disability scales for MTR and MTsat in the cervical spinal cord $\left({ }^{*} \mathrm{p}<0.05\right.$, corrected for multiple comparisons)

\begin{tabular}{|c|c|c|c|c|}
\hline & \multicolumn{2}{|c|}{ EDSS } & \multicolumn{2}{c|}{ T25FW } \\
\hline MTR & $r$ & $\mathrm{p}$ Value & $\mathrm{r}$ & $\mathrm{p}$ Value \\
\hline Median whole SC & -0.228 & 0.104 & -0.055 & 0.700 \\
\hline Median NASC & -0.241 & 0.085 & -0.018 & 0.900 \\
\hline Median SCL & -0.380 & 0.109 & -0.275 & 0.254 \\
\hline Peak height whole SC & -0.465 & $0.001^{*}$ & -0.190 & 0.176 \\
\hline Peak height SCL & -0.199 & 0.415 & -0.281 & 0.243 \\
\hline MTsat & & & & \\
\hline Median whole SC & -0.278 & $0.046^{*}$ & -0.094 & 0.506 \\
\hline Median NASC & -0.333 & $0.016^{*}$ & -0.128 & 0.365 \\
\hline Median SCL & -0.468 & $0.043^{*}$ & -0.378 & 0.110 \\
\hline Peak height whole SC & -0.377 & $0.006^{*}$ & -0.090 & 0.525 \\
\hline Peak height SCL & -0.014 & 0.954 & -0.037 & 0.881 \\
\hline
\end{tabular}

Abbreviations: EDSS = Expanded Disability Status Scale, T25FW = timed 25-foot walk, MTR = magnetization transfer ratio, MTsat = magnetization transfer saturation, $\mathrm{SC}=$ cervical spinal cord, NASC = normal appearing spinal cord, SCL = cervical spinal cord lesions. 


\section{Figure Caption}

Figure 1: Average brain histograms across all subjects of MTsat (a) and MTR (b). The wholebrain MTR histogram is unimodal while the MTsat is multimodal, as a result of the increased contrast between WM and GM. C-spine histograms (c) of MTR and MTsat show similar differences as in the brain. The spinal cord was not further segmented into grey and white matter. Abbreviations: MTR = magnetization transfer ratio, MTsat $=$ magnetization transfer saturation, $\mathrm{WM}=$ white matter, $\mathrm{GM}=$ grey matter, $\mathrm{CSF}=$ cerebrospinal fluid.

Figure 1: MTR and MTsat histograms for the brain and cervical spinal cord.

A

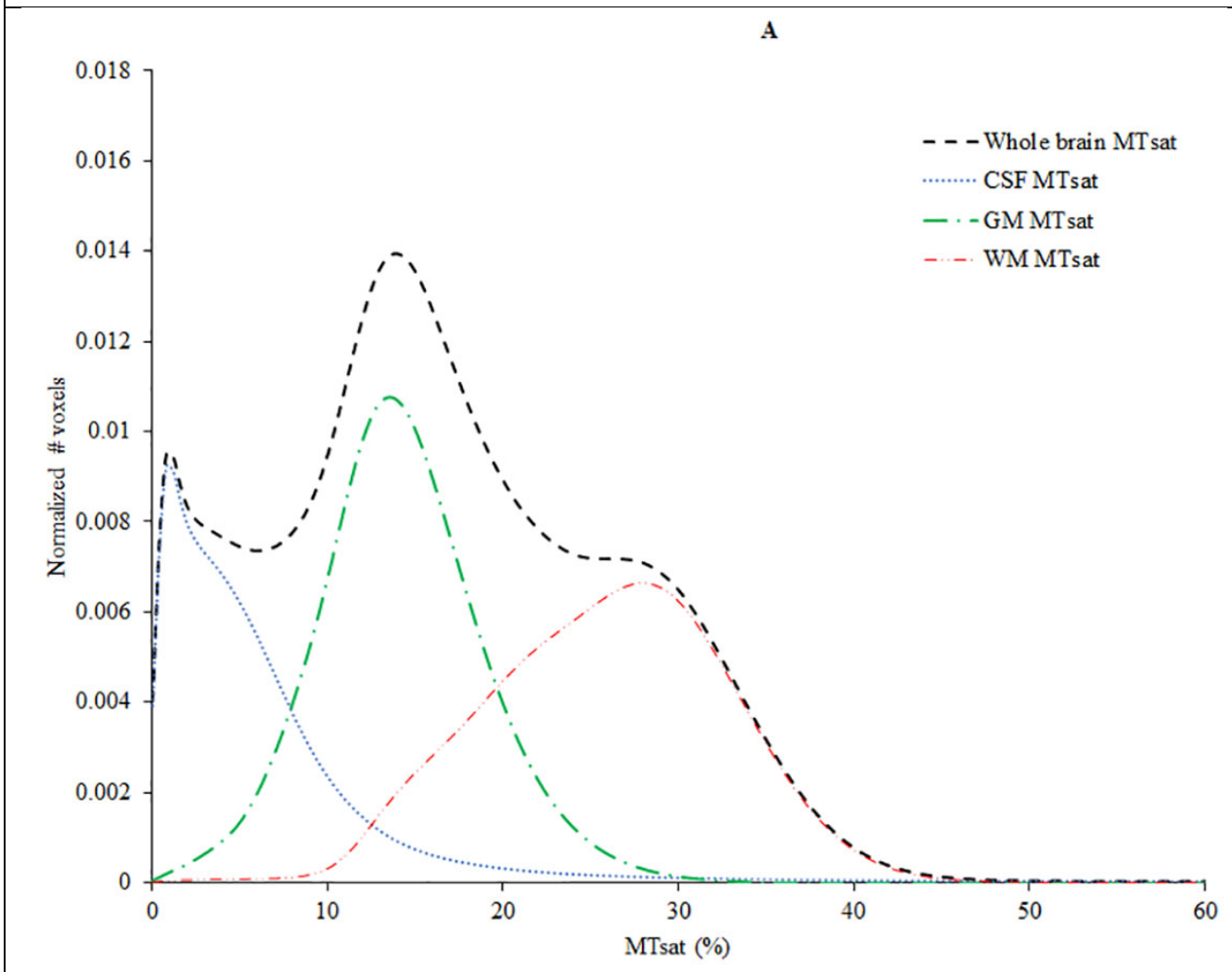




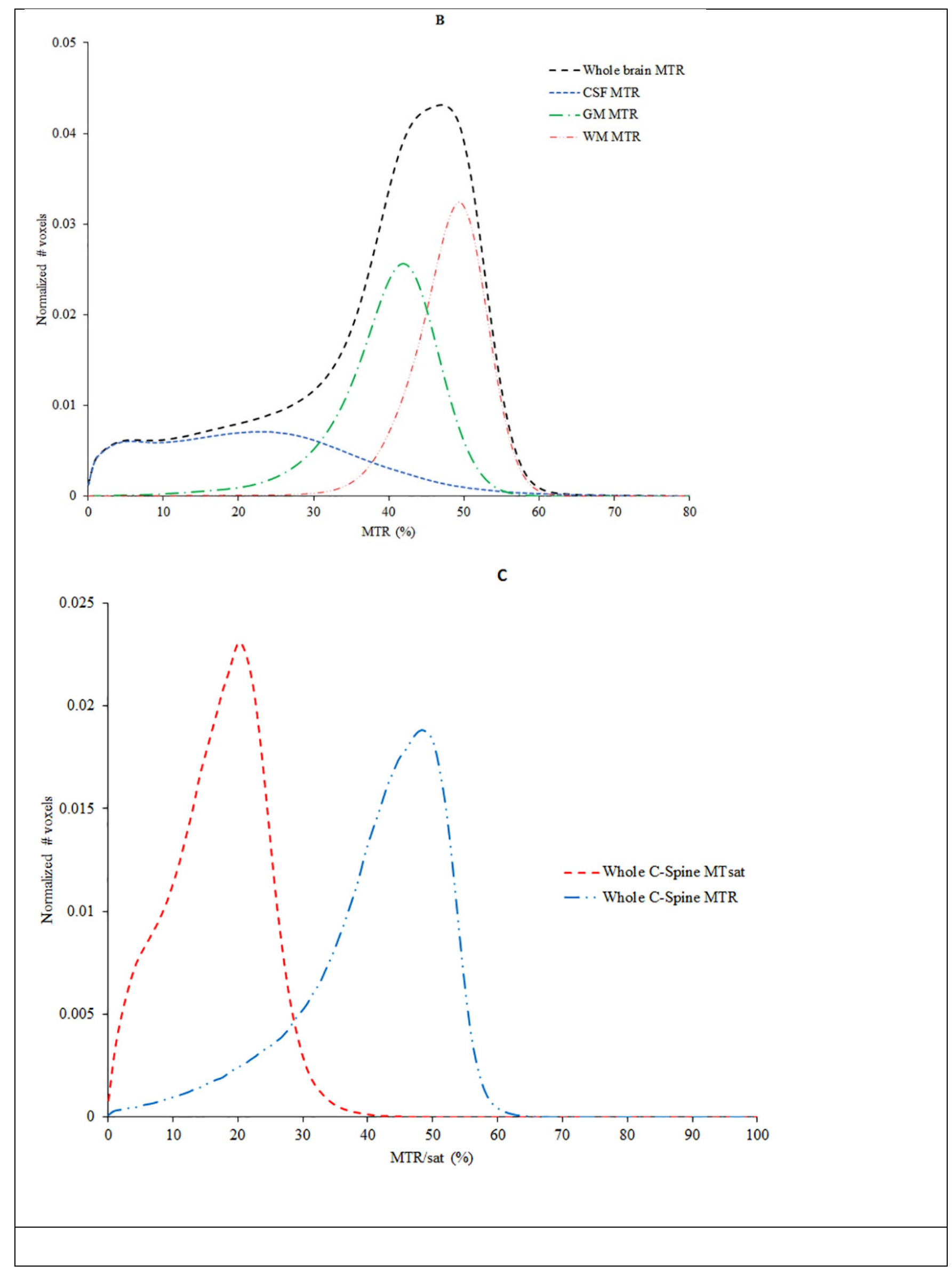


